\title{
ROTATING FLOW AND HEAT TRANSFER IN CYLINDRICAL CAVITIES WITH RADIAL INFLOW
}

\section{Vinod Kumar B. G.}

Post Graduate research student email: vb00052@surrey.ac.uk

\author{
John W. Chew \\ Professor, FRAeS, FASME, CEng \\ Professor of Mechanical Engineering \\ Director of Thermo-Fluid Systems UTC \\ email: j.chew@surrey.ac.uk \\ Thermo-Fluid Systems UTC \\ Faculty of Engineering and Physical Sciences \\ University of Surrey \\ Guildford, GU2 7XH, UK
}

\author{
Nicholas J. Hills \\ Rolls-Royce/Royal Academy of Engineering \\ Reader in Computational Engineering \\ email: n.hills@surrey.ac.uk
}

\begin{abstract}
Design and optimization of an efficient internal air system of a gas turbine requires thorough understanding of the flow and heat transfer in rotating disc cavities. The present study is devoted to numerical modelling of flow and heat transfer in a cylindrical cavity with radial inflow and comparison with the available experimental data. The simulations are carried out with axi-symmetric and 3-D sector models for various inlet swirl and rotational Reynolds numbers upto $2.1 \times 10^{6}$. The pressure coefficients and Nusselt numbers are compared with the available experimental data and integral method solutions. Two popular eddy viscosity models, the Spalart-Allmaras and the $\mathrm{k}-\varepsilon$, and a Reynolds stress model have been used. For cases with particularly strong vortex behaviour the eddy viscosity models show some shortcomings with the Spalart-Allmaras model giving slightly better results than the $\mathrm{k}-\varepsilon$ model. Use of the Reynolds stress model improved the agreement with measurements for such cases. The integral method results are also found to agree well with the measurements.
\end{abstract}

\section{NOMENCLATURE}

$a$ cavity outlet radius, $m$

$b$ cavity inlet radius, $m$

$c$ inlet swirl fraction, $\left(=v_{b} / \Omega b\right)$ $c_{\text {eff }}$ effective inlet swirl fraction

$C_{p}$ non-dimensional pressure difference $\left(=\frac{p_{b}-p}{0.5 \rho \Omega^{2} b^{2}}\right)$

$C_{p, a}$ pressure coefficient $\left(=\frac{p_{b}-p_{a}}{0.5 \rho \Omega^{2} b^{2}}\right)$

$C$ ideal gas specific heat capacity at constant pressure, $\mathrm{J} / \mathrm{kgK}$

$C_{w}$ non-dimensional mass flow rate $(=\dot{m} / \mu b)$

$G$ gap ratio, $(=s / b)$

$H$ stagnation enthalpy, $J$

$k_{a}$ thermal conductivity of air, $W / m K$

$k$ turbulent kinetic energy, $\mathrm{m}^{2} / \mathrm{s}^{2}$

$\dot{m}$ mass flow rate $(\mathrm{kg} / \mathrm{s})$

$N$ number of de-swirl nozzles

$\mathrm{Nu}$ local Nusselt number $\left(=h r / k_{a}\right)$

$n$ exponent in a law defining the gas rotation in the core of vortex chamber

$p$ static pressure, $\mathrm{Pa}$

$p_{\text {ref }}$ static pressure at a reference point, $\mathrm{Pa}$

$P$ gauge pressure, $P a$

$\operatorname{Pr}$ Prandtl number, $\left(=\mu C / k_{a}\right)$

$q$ heat flux, $W / m^{2}$

$Q$ heat transfer to the system, $W$

$r$ radial distance from the axis of cavity, $m$

$r_{s}$ radial location of stagnation point on the cavity end wall (disc), $m$ 
$r_{e}$ radial location where the disc boundary layer entrainment ends, $m$

$R e_{\phi} \quad$ rotational Reynolds number, $\left(\rho \Omega b^{2} / \mu\right)$

$R e_{b}$ rotational Reynolds number for non-rotating cavity, $\left(\rho v_{b} b / \mu\right)$

$s \quad$ axial width of the cavity, $(m)$

$T_{o} \quad$ total temperature, $K$

$T$ temperature, $K$

$u$ radial velocity in the stationary frame of reference, $\mathrm{m} / \mathrm{s}$

$u_{\tau}$ friction velocity, $\mathrm{m} / \mathrm{s}$

$v$ swirl/tangential velocity in the stationary frame of reference, $m / s$

$w$ axial velocity in the stationary frame of reference, $\mathrm{m} / \mathrm{s}$

$x$ non-dimensional radius, $(=\mathrm{r} / \mathrm{b})$

$y$ distance normal to the wall, $m$

$y^{+}$non-dimensional wall distance, $\left(\rho y u_{\tau} / \mu\right)$

\section{Greek}

$\varepsilon$ turbulence dissipation rate, $\mathrm{m}^{2} / \mathrm{s}^{3}$

$\eta$ non-dimensional boundary layer thickness, $z / \delta$

$\delta$ boundary layer thickness on the cavity wall/disc, $m$

$\rho$ density of operating fluid, $\mathrm{kg} / \mathrm{m}^{3}$

$\lambda_{t}$ through flow parametar at cavity inlet, $\left(=C_{w} R e_{\phi}^{0.8}\right)$

$\mu$ molecular viscosity of operating fluid, $\mathrm{Ns} / \mathrm{m}^{2}$

$\bar{v} \quad$ Spalart variable, $\mathrm{m}^{2} / \mathrm{s}$

$\Omega$ angular speed of the cavity, $\mathrm{rad} / \mathrm{s}$

\section{Subscripts}

$a$ value at cavity inner radius

$b$ value at cavity outer radius

$o$ total value in stationary frame of reference

$r$ values at radial location $\mathrm{r}$

rel values in the relative frame of reference

bl values in the boundary layer

$\phi, r, z$ circumferential, radial and axial coordinates

0 values on the disc, $(z=0)$

An overbar '-' values at edge of boundary layer, $(z=\delta)$

\section{Introduction}

Improvements in material properties of gas turbine components and the use of cooling air allow engines to operate at higher pressure ratios and higher gas temperatures thus yielding higher thermal efficiency. The reliance on cooling air makes the secondary air system one of the most critical sections of a gas turbine. The optimum design of these systems maximizes the engine performance and life, and varies depending on the applications (power turbines or jet engines), models and manufacturers. Rotating disc cavities are an important element of engine cooling air systems. As in the review by Owen \& Wilson [1] these disc cavities are broadly classified into rotor-stator, co-rotating and contra-rotating disc cavities. Experimental measurements in geometrically simple cavities have often been used in evaluation of numerical models, and, this approach is adopted here to evaluate various numerical models for the prediction of flow and heat transfer in such cavities with 'radial inflow'. Current industrial practice is to use eddy viscosity turbulence models in CFD modelling of air system flows. The aim of the present work is to provide guidance regarding the validity and limitations of these models in internal flow applications.

Flow visualization experiments in a rotating cavity with radial inflow and outflow in laminar and turbulent conditions were conducted by Owen et al. [2]. The experiments confirmed Hide's [3] isothermal analysis of the source sink flow in a rotating cavity. Hide proposed that the flow consisted of four regions: (i) an inlet source region (ii) thin Ekman type boundary layers, adjacent to the discs, within which all the radial flow occurs (iii) a sink region near to the outlet and (iv) an inviscid core surrounded by source, sink and Ekman layers where the flow is dominantly tangential. Similar flow regimes were observed by Wormley [4] in a narrow vortex chamber experiment when water was injected with high tangential velocity at the outer radius of a stationary cylindrical chamber.

In his experiments, Wormley [4] observed that, when the tangential to radial velocity ratio $\left(v_{b} / u_{b}\right)$ at the inlet was less than 24 , the source region covered the entire chamber and the pressure drop in the cavity could be estimated using the free vortex relations. Further, when the ratio of velocities $\left(v_{b} / u_{b}\right)$ at the inlet was greater than 24, four flow regions, as discussed by Hide [3], appear to form. The pressure distribution in the cavity was dependent only on a particular non-dimensional parameter called the 'modified boundary layer co-efficient' $\left(B L C^{*}\right)$. Flow visualizations showed that the flow entering the chamber was nearly equally split and entrained into the two end wall boundary layers. The radial extent to which this entrainment continues defines the extent of the source region $\left(r>r_{e}\right.$ in Fig 1). In the Ekman layers in the region $r<r_{e}$, the boundary layer thickness slightly decreased with decreasing radius and the inward radial velocity increased. All the radial flow appeared to occur in these thin layers adjacent to the chamber walls. Outside the Ekman layers the flow remained purely tangential and this tangential core spread outward as $B L C^{*}$ increased.

For the rotating cavity applications, Owen et al. [2] proposed a momentum integral technique for the treatment of Ekman boundary layers by extending Hide's [3] work. Solutions were compared with velocity measurements for both radial inflow and outflow cases. Chew and Snell [5] improved the momentum integral solution by including the treatment of disc boundary layers in the source region as entraining layers and developed a method to obtain the radial pressure drop in the rotating cavity with radial inflow. They also used the energy equation to accommodate compressibility effects and demonstrated that for the radial inflow case, with the inlet swirl equal to the rotor speed, 
the non-dimensional pressure drop in a simple cylindrical cavity was dependent only on three parameters: through flow parameter $\left(\lambda_{t}=C_{w} R e_{\phi}^{-0.8}\right)$, the radius ratio for the cavity $(a / b)$ and nondimensional inlet static pressure. It is interesting to note that the non-dimensional parameter given by Wormley [4] and Chew and Snell [5] can be related by $B L C^{*}=0.5 \lambda_{t}{ }^{-5 / 4}$ if $\Omega$ is taken as angular velocity of the fluid at inlet for the stationary cavity.

A brief description of the integral method used in this paper is provided in the next section. Interested readers can also refer to Owen and Rogers [6], Shevchuk [7] and Childs [8] for various developments of integral methods.

Firouzian et al. $[9,10]$ conducted experiments to study the effect of various shroud geometries on the inlet swirl and the cavity heat transfer. These experiments have shown that, the shroud geometry does affect the inlet swirl and hence the radial pressure drop in a rotating cavity. In further experiments, Farthing et al. [11] used de-swirl nozzles at the inlet and Chew at al. [12] used radial fins on one of the discs to reduce the radial pressure drop in a rotating cavity.

Heat transfer measurements with swirling inflow in a wide vortex chamber were reported by Volchkov et al. [13]. In the same paper, a momentum integral approach to estimate the shear stress and heat transfer on the end wall of the chamber was also proposed. Heat transfer measurements with imposed radial inflow in an isothermal rotating cavity were reported by Firouzian et al. $[9,10]$. Average Nusselt numbers for various mass flow rates and rotational Reynolds numbers were reported for various shroud geometries. In another experiment Farthing [14] measured local Nusselt numbers on one disc of the cavity with radial inflow of coolant. Nusselt numbers were calculated based on the measured disc temperatures and heat flux data obtained from the flux meters embedded in the heated disc.

Previous computational fluid dynamics (CFD) and turbulence modelling evaluations for radial inflow in cylindrical cavities are surprisingly limited. Morse [15] reported axisymmet-

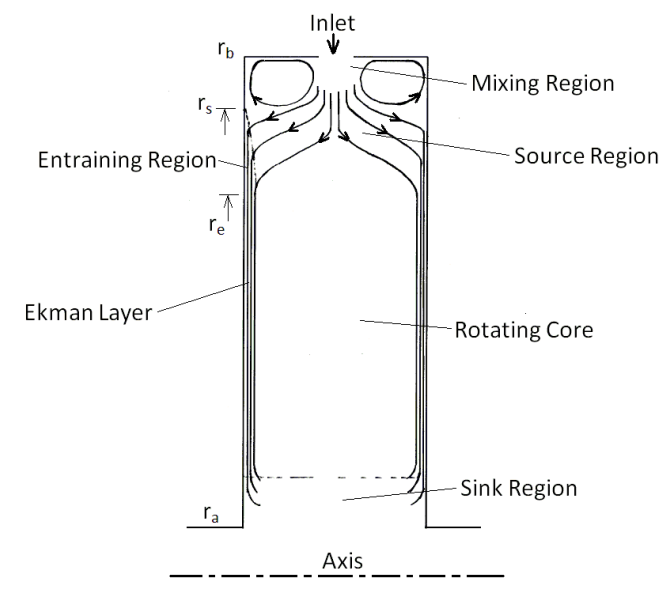

FIGURE 1. Schematic of de-swirled radial inflow in a rotating cavity ric modelling of radial inflow in a rotating cavity using a low Reynolds number $k-\varepsilon$ model. He noticed that due to angular momentum conservation, radial inflow caused higher tangential velocities in the core region which led to severe shearing rates in the Ekman layers. The $k-\varepsilon$ model predicted considerable production of turbulence in these high shear regions, specifically at the lower radii. Another study by Young and Snowsill [16] used the $k-\varepsilon$ model for cooling air off-take optimization studies in the intermediate compressor cavity (IPC) of a three spool aero engine. The primary focus of their study was design optimization of off-take ports. In their validation test case, the CFD predictions of the pressure drop, in the off-take cavity, agreed well with integral method predictions. The present authors are not aware of any further CFD evaluations for super-imposed radial inflow in rotating cavities.

Many of the studies related to turbulence modelling of rotating cavity flows have focused on rotor-stator flows. Early studies by Gosman et al. [17] and Chew [18] used the $k-\varepsilon$ model with axisymmetric approximations. Some researchers have suggested modifications to eddy viscosity models to account for strong rotation. For example, Dacles-Mariani et al. [19] and Spalart and Shurr [20] have suggested modifications to the one equation eddy viscosity model given by Spalart and Allmaras [21] to sensitize the model to rotation and curvature while Torii and Yang [22] and Smirnov and Menter [23] suggested modifications to the two equation eddy viscosity models to achieve similar objectives.

Other researchers, such as Iacovides and Toumpanakis [24], Elena and Schiestel [25] and Chen and Lin [26], have suggested that the Reynolds stress model (RSM) with second moment closure gives good results in body force and rotation dominated flows. On the other hand, standard eddy viscosity models have been used with success for other classes of disc cavity flows by many workers; see, for example, Virr et al. [27]. In a recent paper, Da Soghe et al. [28], compared several two equation eddy viscosity models with Elena and Schiestel's [25] RSM for rotorstator cavities with throughflow. The two equation models gave good agreement for mean flow and an eddy viscosity model was recommended for industrial applications.

In this paper, an integral method, a RSM and two popular eddy viscosity models, Launder and Spalding's [29] two equation $(k-\varepsilon)$ model and Spalart and Allmaras' [21] one equation model (SA) are used to model the flow and heat transfer in vortex chambers and rotating cavities with imposed radial inflow. CFD and integral method solutions are first compared with Wormley [4] and Volchkov et al.'s [13] measurements for narrow and wide vortex chambers respectively. Firouzian et al. [10] and Farthing et al's [14] measurements are then used to test the models for rotating cavity flows and heat transfer.

\section{The Integral Method}

The integral method used here is based on that of von Karman [30] for a disc rotating in a quiescent environment, was de- 
veloped in the 1980s, and is still used in industry. The method and its application to swirling radial inflow between co-rotating plane discs have been reported, for example, by Chew [31], Chew and Snell [5], Chew et al. [12], Farthing et al. [11], but a brief description is included here for completeness. Further descriptions and extensions of the method to rotating cavities with outflow and rotor-stator cavities are given by Chew and Rogers [32], Chew [31] and May et al. [33].

For the present problem, an idealised flow structure is assumed as shown in Fig.1, and treatment of the different regions is summarised below. Note that the sink layer (or exit affected region) is neglected in this treatment.

Momentum integral equations for the disc boundary layers may be derived from mass and momentum balances on a control volume of vanishing radial thickness extending across the boundary layer, or from integration of the equations of motion. Generalising von Karman's analysis for the free disc, the following boundary layer velocity profiles are assumed.

$$
u=u_{1}(r) \eta^{1 / 7}(1-\eta), \quad v=v_{0}+\eta^{1 / 7}\left(\bar{v}-v_{0}\right)
$$

Here $\eta=z / \delta$ where $\delta$ denotes the boundary layer thickness. $\mathrm{u}$ and $\mathrm{v}$ are the radial and tangential velocity components in a cylindrical coordinate system $(r, \phi, z)$, with the disc at $z=0$ and the axis of rotation at $r=0 . u_{1}$ is a function of $r$ only, $v$ is the tangential velocity component with subscript 0 and the overbar denoting values at the disc $z=0$ and at the boundary layer edge $z=\delta$, respectively.

Following Chew and Snell [5], the momentum integral equations for the disc boundary layers are as follows.

$$
\begin{gathered}
I_{2} \frac{d\left(\rho r u_{1}^{2} \delta\right)}{r d r}+I_{4} \frac{2 \rho \delta v_{0}\left(\bar{v}-v_{0}\right)}{r}+I_{5} \frac{\rho \delta\left(\bar{v}-v_{0}\right)^{2}}{r}= \\
\frac{u_{1}}{\left(v_{0}-\bar{v}\right)} 0.0225\left(\frac{\mu}{\rho \delta}\right)^{1 / 4} \rho\left(v_{0}-\bar{v}\right)\left[u_{1}^{2}+\left(v_{0}-\bar{v}\right)^{2}\right]^{3 / 8}
\end{gathered}
$$

and,

$$
\begin{gathered}
I_{1} \frac{\left(v_{0}-\bar{v}\right)}{r} \frac{d\left(\rho r u_{1} \delta\right)}{d r}+I_{1} \frac{\rho u_{1} v_{0} \delta}{r}+I_{1} \rho u_{1} \delta \frac{d v_{0}}{d r}+ \\
I_{3} \frac{d\left[\rho r^{2} \delta u_{1}\left(\bar{v}-v_{0}\right)\right]}{r^{2} d r}= \\
0.0225\left(\frac{\mu}{\rho \delta}\right)^{1 / 4} \rho\left(v_{0}-\bar{v}\right)\left[u_{1}^{2}+\left(v_{0}-\bar{v}\right)^{2}\right]^{3 / 8}
\end{gathered}
$$

where,

$$
I_{1}=\frac{49}{120} \quad I_{2}=\frac{343}{1656} \quad I_{3}=\frac{49}{144} \quad I_{4}=\frac{1}{8} \quad I_{5}=\frac{2}{9}
$$

In these equations $\rho$ and $\mu$ denote fluid density and viscosity. The terms on the right-hand-sides of equations (2) and (3) represent shear stresses on the disc and are, again, generalisations of von Karman's assumptions.

An integral equation for energy conservation is used to estimate disc heat flux and fluid temperature. The derivation of this equation follows that of Chew and Rogers [32] who considered heat transfer for radial outflow between co-rotating discs and Chew and snell [5] who considered adiabatic flow. A boundary layer profile for the stagnation enthalpy is assumed as follows

$$
H=H_{0}+h(\eta, r)\left(\bar{H}-H_{0}\right)
$$

Integration of the energy equation across the boundary layer then gives

$$
\begin{gathered}
I_{1} \frac{\left(H_{0}-\bar{H}\right)}{r} \frac{d\left(\rho r u_{1} \delta\right)}{d r}+I_{1} \rho u_{1} \delta \frac{d H_{0}}{d r}+ \\
\frac{d\left[\rho r \delta u_{1}\left(\bar{H}-H_{0}\right) I_{6}\right]}{r d r}= \\
q_{0}+0.0225\left(\frac{\mu}{\rho \delta}\right)^{1 / 4} \rho v_{0}\left(v_{0}-\bar{v}\right)\left[u_{1}^{2}+\left(v_{0}-\bar{v}\right)^{2}\right]^{3 / 8}
\end{gathered}
$$

where,

$$
I_{6}=\int_{0}^{1} \eta^{1 / 7}(1-\eta) h(\eta, r) d \eta
$$

In the solutions presented here $I_{6}$ and the disc heat flux $q_{0}$ are determined following the "second method" described by Chew and Rogers [32]

Referring to Fig 1, the disc boundary layer can be divided into three regions; the boundary layer outflow region $r>r_{s}$, the entraining inflow region $r_{e}<r<r_{s}$, and the non-entraining inflow region $a<r<r_{e}$. Here the inlet swirl fraction $\left(c=v_{b} / \Omega b\right)$ is assumed to be between 0 and 1. The stagnation point on the disc at $r=r_{s}$ is calculated as the point at which the swirl velocity in the core equals the disc speed. The point at which all the inflow is entrained into the boundary layer $\left(r=r_{e}\right)$ is determined from the solution of the integral equations.

\subsection{Inviscid flow regions}

Outside the boundary layers and the mixing region viscous effects are neglected and approximate analytical solutions are used to describe the flow. In the source region these are the free vortex relationship and the assumption of constant stagnation enthalpy. In the central core rotational effects are assumed to dominate, with symmetry about the central axial plane, so that the axial and radial velocity components are zero, and the axial 
gradient of tangential velocity is zero. The stagnation enthalpy is also assumed independent of axial position in the core, with the integral energy equation used to estimate the variation of stagnation enthalpy with radius.

With the usual boundary layer assumptions, pressure is a function of radius only. This is estimated using the radial equilibrium equation for the core which reduces to a balance of the pressure gradient with the centrifugal force. With pressure estimated from this equation and temperature calculated from the core stagnation enthalpy and velocity the fluid properties may be obtained using an equation of state and appropriate correlations.

\subsection{Shroud and mixing region}

The mass flow in the thin boundary layer on the shroud is assumed to be constant and equal to that in the outflow boundary layer on the disc at $r=b$. A friction factor is also calculated for the disc boundary layer at $r=b$ and this is used to estimate the change in bulk tangential velocity as the fluid flows along the shroud to the mixing region. The shroud boundary layer flow and the inflow with swirl fraction $c$ are assumed to fully mix in the mixing region, and an effective inlet swirl fraction $\left(c_{e f f}\right)$ is calculated for use in calculation of the inviscid flow region. A similar treatment could be applied to the energy balance to account for heat transfer. However, the heat transfer test cases considered in this paper do not require inclusion of a mixing region.

\subsection{Numerical solution}

Solutions of the integral equations and auxiliary relations were obtained with a standard mathematical library routine for ordinary differential equations. The computer program used was developed from that of Chew and Rogers [32]. The solutions for both the outflow and inflow regions of the layer were started at the stagnation point $r=r_{s}$. Some iteration of solutions was required to find the effective inlet swirl fraction, $c_{e f f}$.

\section{CFD modelling}

For most of the CFD models a thin $3 D$ sector domain was used with rotationally periodic boundary conditions. ANSYS ICEMCFD software was used for mesh generation. To obtain a good quality hexahedral mesh in the thin sector, the axis was avoided by inserting a thin central shaft with radius of 2 to $3 \%$ of the cavity radius. CFD solutions were obtained using RollsRoyce plc.'s in-house finite volume coupled solver Hydra and the commercial solver FLUENT 6.3, with second order discretization. Details of the Hydra solver are given in Moinier [34]. The $k-\varepsilon$ model in its standard form was used in both solvers. The modified Spalart-Allmaras (SA) model with vorticity and strain based production, proposed by Mariani et al. [19], was used in both solvers. For simulations using the RSM model with wall functions in FLUENT, the default wall boundary conditions (as described in the FLUENT user guide [35]) were used for the Reynolds stresses. This default setting is to calculate the values of the Reynolds stresses at the near wall nodes from the value of k. While using the RSM model with enhanced wall treatment (i.e. using a one equation model close to the walls), this default setting caused convergence difficulties for some cases. The alternative option of setting the near wall Reynolds stresses from the wall shear stress was therefore used for the RSM with enhanced wall treatment cases. As all these cases had near wall mesh y+ values less than 2 , and hence the one equation model was actually being used for the near wall nodes, this change should have negligible effect on the converged solution, and this was checked for one test case.

The inlet turbulent intensity and length scale for all the simulations were assumed as $2 \%$ and $1 \mathrm{~mm}$ respectively. Using a turbulent intensity of $10 \%$ and length scale of $7 \%$ of hydraulic diameter, when using the $k-\varepsilon$ model and RSM in FLUENT and the SA model in Hydra, for test cases S1 and R1 had negligible effect on final solutions. In most of the cases the mesh $y^{+}$values near the walls were in the range of 30 to 120 and standard wall functions were used. For near-wall resolved models, the nearwall mesh $y^{+}$values were below 2 in the cavity.

While solving in Hydra, a multigrid approach was used with four levels of grids and a density based solution algorithm. The pressure based approach was adopted while simulating the cases in FLUENT. Mesh dependency was checked by changing the grid points in all three directions (axial, radial and tangential) and comparing the velocity and pressure distributions along specified lines in the cavity. Convergence of the solutions was assumed when the residuals were below the specified tolerance and mass flow rate and wall torques reached steady values with respect to the iterations. Overall angular momentum and energy balances were also checked for all solutions.

\section{Flow and heat transfer in a stationary cavity}

In the problems considered here flow radially enters the stationary cylindrical cavity at the outer radius $(b)$ with a swirl component of velocity $v_{b}$ and exits axially at the inner radius $(a)$. As discussed in the introduction, with the superimposed radial inflow and sufficient inlet swirl, four flow regions form in both stationary and rotating cavities. Chew and Snell [5] successfully used the integral method, developed for the rotating disc cavities, to estimate the pressure drop in a stationary cylindrical cavity thus confirming the experimental observations. In this paper, the CFD modelling of radial inflow in a stationary cavity is undertaken first and performance of various turbulence models is considered. Flow in a narrow cylindrical cavity $(G=0.286)$ is discussed in Section 4.1. Computations of flow and heat transfer in a wide cylindrical cavity $(G=2.0)$ are presented in Section 4.2.

\subsection{Test case S1}

4.1.1 Formulation The experiments by Wormley [4] in a narrow vortex chamber $(s=0.0254 \mathrm{~m}, b=0.0889 \mathrm{~m}$, $a=0.00635 \mathrm{~m}$ ) with high inlet swirl were used to evaluate turbu- 


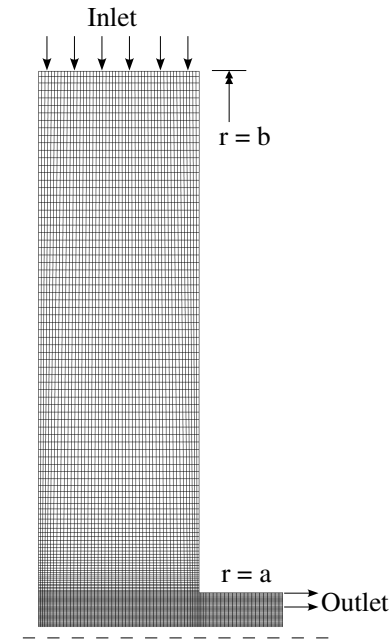

FIGURE 2. Mesh used for test case S1

lence models for radial inflow in a stationary cavity with small gap ratio $(G=0.2857)$. When measuring pressures, Wormley used air as the working fluid, with pressure tappings in the stationary housing.

A sector geometry with one degree sector angle was used for these simulations. The axisymmetric approximation to the experimental geometry and CFD mesh used are shown in Fig 2. The fluid enters the cavity uniformly through the outer cylindrical surface with strong swirl and flows out of the cavity through an extension pipe at the inner radius. The case with $v_{b} / u_{b}=59.8$ was computed using SA and $k-\varepsilon$ models in Hydra and FLUENT and also using the RSM model available in the latter solver. Mesh dependency of solutions was investigated by comparing solutions obtained on two meshes with 8000 and 22,000 cells. This confirmed that an acceptable degree of mesh independence was achieved. Near-wall layer resolved solutions were obtained on a mesh with 62,500 cells.

In his experiments, Wormley defined the inlet radial velocity as $u_{b}=\dot{m} /(2 \pi \rho s b)$ and tabulated its value for various inlet conditions. For $v_{b} / u_{b}=59.8$, this area averaged inlet radial velocity was $0.2438 \mathrm{~m} / \mathrm{s}$. Corresponding values of inlet rotational Reynolds number $\left(\operatorname{Re}_{b}=\rho v_{b} b / \mu\right)$ and non-dimensional mass flow rate $\left(C_{w}=\dot{m} / \mu b\right)$ were 93,600 and 2800 respectively. Air was taken as the working fluid in these simulations. As the absolute pressure ratio across the chamber was close to unity $\left(p_{b} / p_{a} \approx 1.06\right)$, the non-dimensional results approximate those for any incompressible fluid at the values of $C_{w}$ and $R e_{b}$ given.

4.1.2 Results The radial pressure distribution on the left hand wall of the chamber is shown in Fig 3. Among the tested eddy viscosity models, predictions from the SA model in Hydra agreed best with the pressure measurements. Differences between results from the two solvers while using SA model are mainly attributed to different implementation of wall functions.
When the simulations were carried out resolving the near wall layer using the SA model, the Hydra results (Fig 3(a)) moved slightly farther from the previous predictions (using wall function) while the predictions from FLUENT (Fig 3(c)) slightly improved. Other workers have also found similar sensitivity to wall function implementation; see for example Javiya et al. [36].

The predictions from the integral method were obtained assuming a very slow rotation of the cavity and agree well with the measured values at all radii as shown in Fig 3(b). The FLUENT results for the two-layer $k-\varepsilon / k-1$ model show negligible difference from the wall-function $k-\varepsilon$ model as shown in Fig 3(d). The RSM model in FLUENT (Fig 3(e)) agreed well with the measurements and showed no sensitivity to wall treatment.

The experiments show that for $(1-r / b)>0.7$ the radial pressure gradient increases sharply due to the formation of a strong vortex. As shown in Fig 3(b \& d), the $k-\varepsilon$ model in both solvers fails to predict this sharp pressure gradient (proportional to $\rho v^{2} / r$ ) caused by the highly swirling confined vortex near the inner radius of the cavity. This is attributed to excessive turbulent viscosity estimated by this model at lower radii of the cavity as
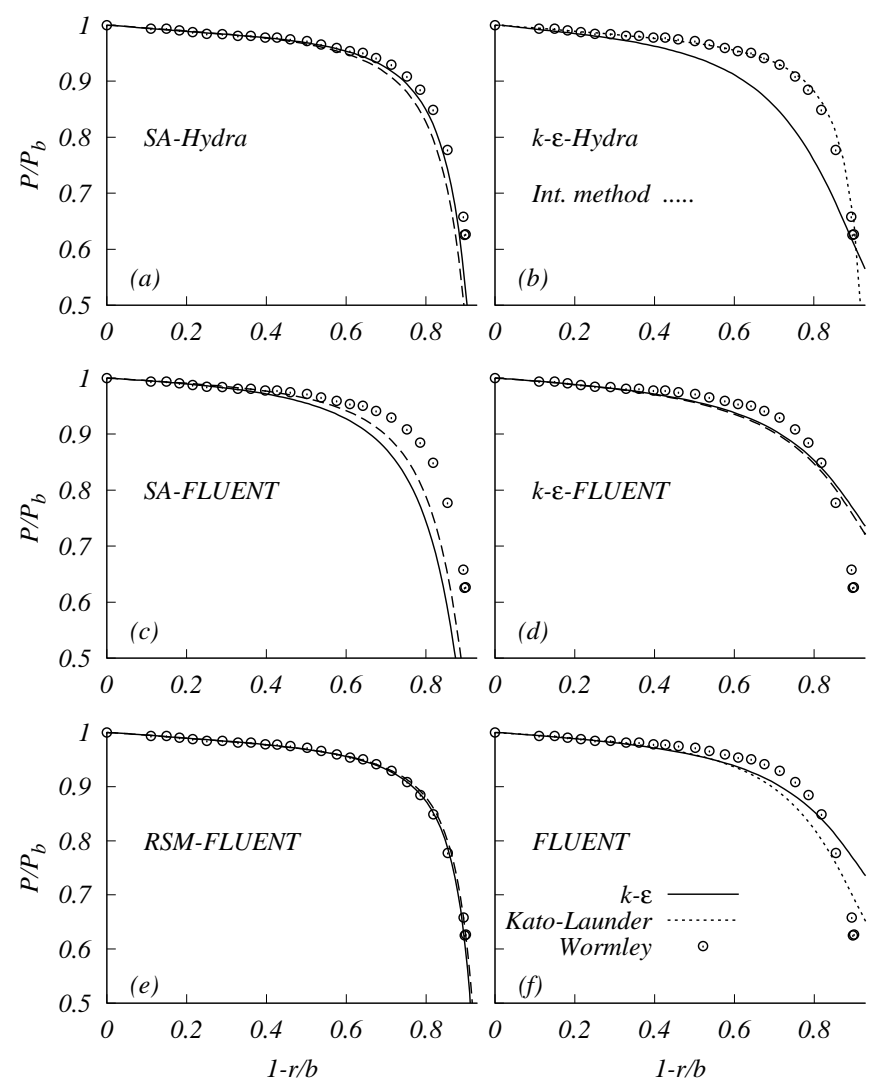

FIGURE 3. Radial pressure distribution on the left end wall of vortex chamber for test case $S 1\left(C_{w}=2800, R_{b}=93600\right)$. - Wall functions, - - - enhanced wall treatment, 'o' Wormley's [4] experiments. Note that the vertical axis represents ratio of local gauge pressure to inlet gauge pressure as presented by Wormley [4] 


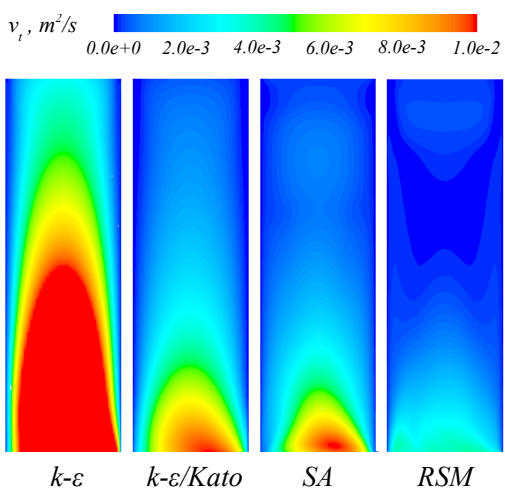

FIGURE 4. Turbulent viscosity contours predicted by four turbulence models in test case $\mathrm{S} 1$

shown in Fig 4.

In the RSM model the turbulent viscosity, shown in Fig 4, is calculated using $v_{t}=C_{\mu} k^{2} / \varepsilon$ where $C_{\mu}=0.09$. This is identical to the eddy viscosity relation in $k-\varepsilon$ model but the kinetic energy is obtained by taking the trace of Reynolds stress tensor.

A variant of the $k-\varepsilon$ model applying the Kato-Launder modification to the turbulent production term in the k-equation with wall functions was also tested. The results are shown in Fig 3(f). Compared to the basic $k-\varepsilon$ model, the Kato-Launder modification showed a little steeper pressure gradient at lower radius of the cavity but was hardly nearer to the measurements. The modification reduced the turbulent viscosity in the core region compared to the $k-\varepsilon$ model but this was still considerably higher than for the SA and RSM models, as shown in Fig 4. It is to be noted that among all the models considered the RSM predicted least turbulent mixing in the rotating core region. The good agreement of results from this model and integral method (which assumes the rotating core to be inviscid) with the measurements suggests that the RSM model performed better than other models directly because it reproduces the suppression of turbulence by rotational effects in the core region. Success of RSM model in estimating anisotropic Reynolds Stresses in rotating flows has been reported by many investigators such as Howard et al. [37], Chen and Lin [26], Shur et al. [38], Iaccarino et al. [39] and Poncet et al. [40].

\subsection{Test case S2}

Volchkov et al. [13] carried out experimental and theoretical investigations of flow and heat transfer in a wide vortex chamber $(G=2.0)$. The chamber dimensions were $b=0.1 \mathrm{~m}, a=0.015 \mathrm{~m}$ and $s=0.2 \mathrm{~m}$. Coolant (air) was supplied to the chamber through 4 rows of nozzles, which were mounted tangentially to the outer periphery of the chamber. Each row had 10 equi spaced nozzles along the axial span of the chamber. An axial pipe of $0.015 \mathrm{~m}$ radius attached to one of the chamber walls was used as an outlet. Laser-Doppler anemometry was used for velocity measurement in the chamber. For heat transfer experiments a thin circumferentially slotted copper foil, coated on the inner face of the side

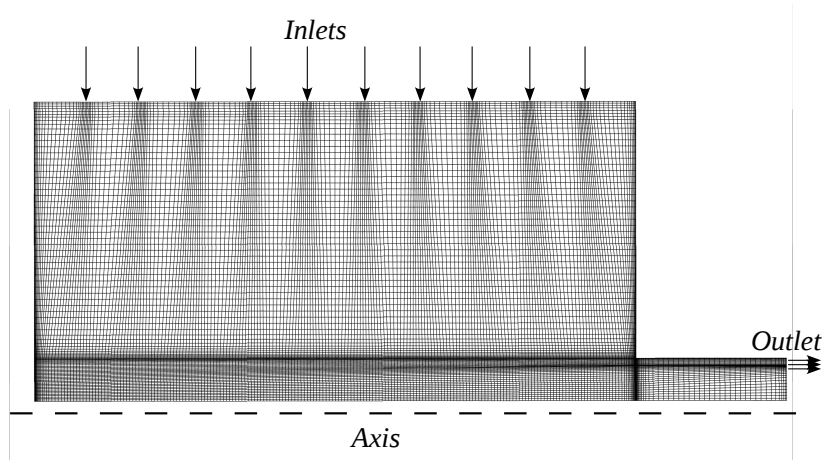

FIGURE 5. Mesh used for test case S2

wall opposite to the chamber outlet, was used as the heating surface. The outer surfaces of the chamber walls were thermally insulated. Volchkov et al. [13] quoted the heat transfer errors during the experiments at less than $10 \%$. The temperature measurements on the side wall were done using 16 thermocouples fitted in the space between the concentric copper foils. Temperature measurements at the axial mid-plane were done using a single thermocouple which was traced over the entire diameter.

To avoid the axis in the computation domain, a narrow shaft of $0.001 \mathrm{~m}$ radius was introduced at the centre of the chamber. Two cases have been studied for the evaluation of flow and heat transfer in wide vortex chambers. The case with inlet Reynolds number $R e_{b}=0.22 \times 10^{5}$ and Rossby number (defined by [13] as $R_{o}^{\prime}=\dot{m} /\left(v_{(r / b=0.95)} b^{2}\right)$ of 0.1 was used as test case for flow studies and the case with $R e_{b}=2.0 \times 10^{5}$ and $R_{o}^{\prime}=0.07$ was used for the heat transfer studies. The non-dimensional mass flow rate $\left(C_{w}\right)$ for these cases were 2,360 and 14,000 respectively. It should be noted that the tangential velocity used to calculate the Rossby number was measured at the outer edge of the shroud boundary layer (at $r / b \approx 0.95$ ).

4.2.1 Formulation A one degree sector domain with 97,000 hexahedral cells was used for computations with standard wall functions and a 0.4 degree sector with 690,000 cells was used to simulate the cases resolving near wall regions. 10 parallel circumferential slots, each having an area equal to the area of intersection of four nozzles with the peripheral shroud, were used as inlets for the CFD domain as shown in Fig 5. The inlet conditions were determined assuming total pressure of $101300 \mathrm{~Pa}$ and total temperature of $290 \mathrm{~K}$. The inlet velocity angles were adjusted to achieve the required tangential velocity at the edge of shroud boundary layer. The outlet pressure was adjusted to achieve the desired mass flow rate. For the first test case all the walls were assumed to be adiabatic. For the second test case involving heat transfer calculations, a temperature profile was specified on the side wall opposite to the outlet and an isothermal boundary condition was assumed on the other side wall as this all was not insulated and the reported temperature distribution was almost constant at $290 \mathrm{~K}$. The temperature pro- 


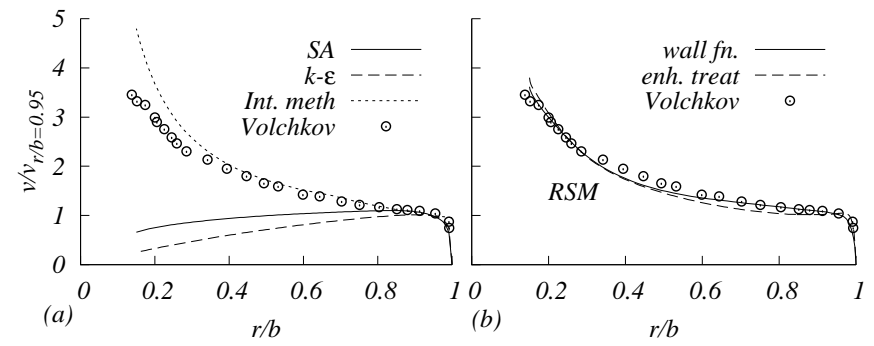

FIGURE 6. The tangential velocity profile at the axial mid-plane of the cavity for test case $S 2\left(\operatorname{Re}_{b}=22000, C_{w}=2360\right)$. Results using (a) eddy viscosity models in Hydra and integral method (b) RSM model in FLUENT

file was obtained from the experimental measurements reported by Volchkov et al. [13]. The inlet shroud, central shaft and exit pipes were assumed to be adiabatic. The overall angular momentum balance error in the converged solution was was less than $3 \%$ and $1 \%$ of the difference between inlet and outlet angular momentum fluxes for the cases using wall functions and near wall resolved models respectively.

4.2.2 Results Simulations were undertaken using the $k-\varepsilon$ and SA models in the Hydra solver using standard wall functions. Further simulations, resolving the near wall regions, were carried out using the SA model in Hydra and RSM model in FLUENT on a finer mesh. In the RSM model near-wall Reynolds stresses were specified in terms of the wall shear stress as described in Section 3. Solutions were again obtained using the integral method by assuming a very slow rotation (of the order of $0.01 \mathrm{rad} / \mathrm{s}$ ) of the cavity.

Non-dimensional tangential velocity profiles at the axial mid plane (at $z=0.1 \mathrm{~m}$ ) of the cavity for the flow test case are shown in Fig 6. The tangential velocity at $x=0.95$ was used to non dimensionalize the velocity profiles. Both the eddy viscosity models failed to match the measured values. Due to excessive turbulent viscosity in the core region, the vortex core did not appear to form in the simulations using SA and $k-\varepsilon$ models. If the tangential velocity in the vortex is approximated by the power law $v / v_{b} \approx 1 / x^{n}$ with $n=1$ representing free vortex, then both the eddy viscosity models predicted nearly forced vortex flow ( $n \approx-0.4$ by SA model and $n \approx-1$ by $k-\varepsilon$ model) in the cavity while the vortex behaviour reported in the experiments was closer to a highly swirling free vortex $(n \approx 0.7)$. The RSM model results, using the wall functions as well as a near wall resolved treatment, matched the measurements reasonably well and also appeared to resolve the inner core region. However, the integral method solution predicted higher swirl velocities at the lower radius of the cavity than the experiment.

In the second experiment considered, Volchkov et al. [13] carried out heat transfer studies. Temperature and heat transfer measurements were made on the side wall opposite to the outlet vent. The total temperature at the axial mid-plane of the cham-
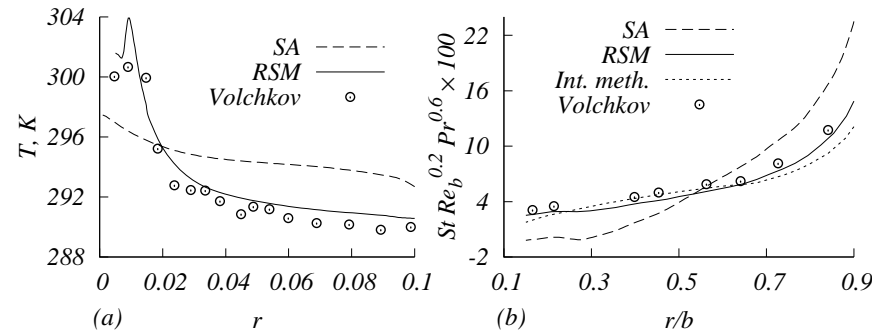

FIGURE 7. (a) Total temperature at the axial mid plane of the cavity (b) Local Stanton number on the side wall opposite to the outlet vent at $\left(R e_{b}=200,000\right.$ and $\left.C_{w}=14,000\right)$ for test case $S 2$

ber and the local Stanton number on the heated side wall of the chamber are plotted in Fig 7 (a) and (b) respectively. Volchkov et al. [13] defined the Stanton number as $S_{t}=q_{0} /\left(\rho C u_{m}\left(T_{0}-\bar{T}\right)\right)$ (where $q_{0}$ denotes wall heat flux and $T_{0}$ and $\bar{T}$ are temperatures on the wall and edge of the boundary layer). The quantity $u_{m}$ in the above equation denotes the maximum radial velocity in the boundary layer estimated by Volchkov et al. [13] and is given by $u_{m}=v_{b}\left[0.4\left((r / b)^{-2}-1\right) / n\right]^{0.5}$, where $n \approx 1$. Only the solutions computed from enhanced wall treated models are reported $(k-\varepsilon$ model in Hydra excluded). Predictions from RSM are closer to the measurements than the SA model in Hydra. The Stanton numbers calculated using the integral method are also close to the measurements in the inner part of the cavity but show significant differences for $r / b>0.7$.

\section{Radial inflow in a rotating cavity}

In the cases considered here, cavities were formed by two co-axial discs and a peripheral shroud all rotating at the same speed. Fluid entered this co-rotating cavity at the outer radius $b$ and left the cavity at the inner radius $a$. Depending on inlet swirl $c, C_{w}$ and $R e_{\phi}$, four distinct flow regions as shown in Fig 1 and discussed in Section 1 may form. Firouzian et al. [9, 10] concluded that the effective inlet swirl (c) depends primarily on the shroud geometry and whenever its value was less than unity a small region of radial outflow was observed near the shroud. They also found that for a given inlet total temperature and total pressure, the radial pressure drop in a co-rotating cavity mainly depended on the radius ratio $(a / b)$, inlet swirl $(c), C_{w}$ and $R e_{\phi}$ which was later supported by Farthing [14] and Farthing et al. [11]

Computations of radial inflow in a narrow co-rotating cavity with unit inlet swirl is summarized in test case $R l$, flow in a cavity with de-swirled flow is discussed in test case $R 2$ and heat transfer in a co-rotating cavity with unit inlet swirl is considered in test case $R 3$.

\subsection{Test case R1}

Firouzian et al. $[9,10]$ conducted experiments to study the flow and heat transfer in a narrow rotating cavity with super- 
imposed radial inflow. The radius ratio $a / b$ and gap ratio $G$ were 0.1 and 0.134 respectively with the outer radius being $0.381 \mathrm{~m}$. To study the effect of $C_{w}$ and $R e_{\phi}$ and effective inlet swirl $c$ on the flow, they used six different shrouds and varied the flow and rotation speed of the cavity. Tangential velocity was measured in the axial mid plane at various radial locations in the cavity using Laser Doppler Anemometry. From the studies, Firouzian et al. [9] concluded that the inlet swirl fraction was less than unity $(c \approx 0.42$ to 0.72 ) for shrouds with a series of holes or a continuous slot and $c=1$ for a shroud with foam filled slot. They also concluded that it is difficult to accurately predict the effective inlet swirl for a given cavity as it greatly depended on the shroud geometry.

5.1.1 Formulation The case with inlet swirl $c=1$ was selected for numerical studies. This corresponds to an experiment in which the inflow passed through rotating foam filled slot. The flow domain consisted of a $2^{\circ}$ sector of the cavity, similar to test case $\mathrm{S} 1$ with an axial extension pipe attached to the outlet. The central shaft used in the experiments had a radius of 0.0125 $m$. The inlet shroud had a narrow slot of width $5 \mathrm{~mm}$ at the axial mid-plane of the cavity. For mesh dependency studies, the mesh sizes were varied from 11,500 cells for a coarse mesh to 41,500 for a fine mesh. The coarser mesh had at least $30 \%$ less nodes in the axial, radial and tangential directions than the finer mesh. A relative frame formulation was used with the coordinate system (and all boundaries) rotating at the angular velocity of the cavity. At the inlet boundary, total pressure $(101,300 \mathrm{~Pa})$, total temperature $(300 \mathrm{~K})$, turbulence intensity $(2 \%)$, turbulence length scale $(1 \mathrm{~mm})$ and inlet velocity vector (normal to the boundary) were specified. For the SA model a turbulent to molecular viscosity ratio of 10 was assumed at the inlet. At the outlet boundary, pressure was varied to achieve the target mass flow rate.

The test case considered had a rotational Reynolds number of $R e_{\phi}=3.45 \times 10^{5}$ and non-dimensional mass flow rate of $C_{w}=1,300$. All the walls were considered to be adiabatic. The overall angular momentum imbalance (the difference between change in angular momentum flux of the fluid in the cavity and the total wall torque) was less than $2 \%$ of the total change in angular momentum flux in the cavity for all the computations.

5.1.2 Results Simulations were run in Hydra and FLUENT using SA and $k-\varepsilon$ models with standard wall functions. The non-dimensional absolute velocities at the axial midplane of the cavity are shown in Fig 8. Among the Hydra results (Fig 8.a), the SA model showed promising agreement with measurements while the $k-\varepsilon$ model results deviated from the measurements at the lower radius of the cavity as observed in test case S1. In the FLUENT solver both the eddy viscosity models showed good agreement with the measurements (Fig 8.b) and the RSM model showed the closest match. However at lower radii, where the rotation effects were strongest, the re-

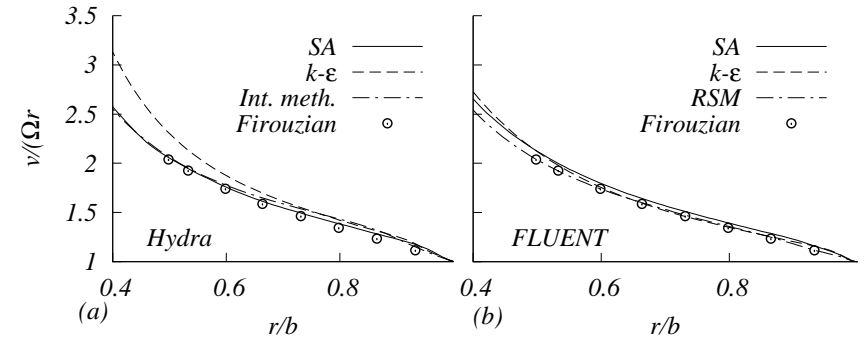

FIGURE 8. The non-dimensionalized tangential velocity profile at the axial mid-plane of the cavity for test case $R 1\left(R_{\phi}=3.45 \times 10^{5}, C_{w}=\right.$ 1300). Results from turbulence models in (a) Hydra and integral method (b) FLUENT solver

sults showed some variations for different turbulent models. No velocity measurements were available at lower radii to validate these results. The solutions obtained from the momentum integral method matched the measurements closely.

\subsection{Test case $\mathbf{R 2}$}

In this test case the effect of de-swirling the flow at the inlet of a rotating cavity is considered in comparison with the experiments reported by Farthing et al. [11,14]. To reduce the absolute tangential velocity of the incoming fluid, Farthing et al. [11] used de-swirl nozzles. The nozzles were connected to an upstream settling chamber to which the inlet air enters through a foam filled slot. This arrangement was incorporated to ensure uniform entry of air into the cavity through the nozzles and for accurate prediction of inlet swirl. The axes of the nozzles were located in the $r-\phi$ plane and made an angle of $30^{\circ}$ with the shroud tangent at the point of intersection of the axes with the shroud. For a given cavity rotational speed the effect of changing $C_{w}$ and $c$ on the cavity pressure drop was studied. In the experiments, static pressure taps at $r=172,223,274$ and $325 \mathrm{~mm}$ were drilled on the right hand disc shown in Fig 9.

Two cases are presented here. In the first case $R 2.1$, local $C_{p}$ distributions predicted by various turbulence models on one of the discs are compared with the data for two different inlet swirl (c) conditions. The experimental set-up considered for this case had two circumferential rows, each containing 20 equally spaced de-swirl nozzles.

In the second case study, $R 2.2$, CFD predictions of cavity pressure drop co-efficient $C_{p 0}=\left(p_{b}-p_{x=0.51}\right) /\left(0.5 \rho \Omega^{2} b^{2}\right)$ for the range of $C_{w}$ and $c$, listed in Table 1, at a given rotational Reynolds number $\left(R e_{\phi}=0.61 \times 10^{6}\right)$ are compared with the experimental data. This case is interesting because of the exhibition of an "S-curve" flow characteristic as explained by Farthing et al. [11] ie., for a given $C_{p 0}$ value, there may be more than one possible flow rate. The geometry was different from $R 2.1$ with two circumferential rows housing 40 nozzles each.

5.2.1 Formulation Both axisymmetric and $3 D$ simulations were undertaken for test case $R 2.1$ and only $3 D$ modelling 


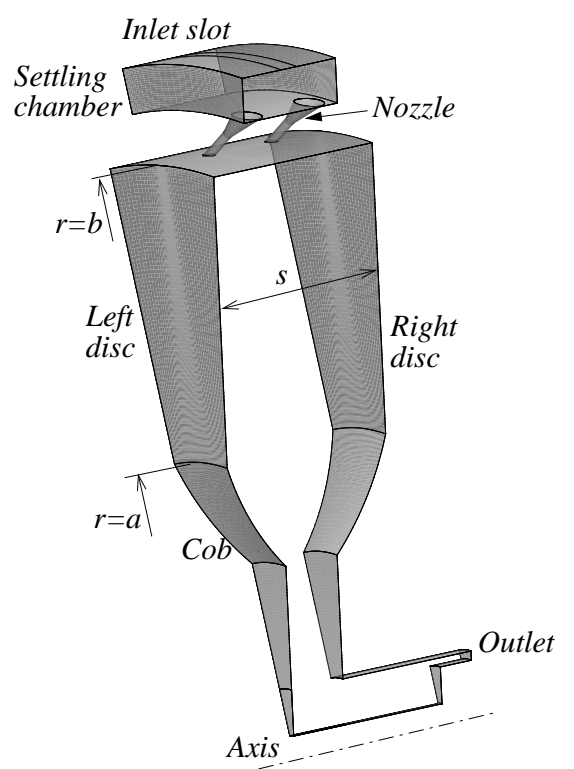

FIGURE 9. 3D geometry used for test case R2.1

for $R 2.2$. For the axisymmetric modelling a $2^{\circ}$ sector geometry was constructed. For $3 D$ modelling in $R 2.1$ an $18^{\circ}$ sector of the cavity including the settling chamber and a pair of nozzles at the inlet was constructed. For case $R 2.2$ the $18^{\circ}$ sector model consisted of 4 nozzles, two on each row, without the settling chamber. The $3 D$ geometry used for this test case is shown in Fig 9. More details about the experimental set up can be found in Farthing et al. [11]. The cavity starts at $r=0.33 \mathrm{~m}$ and ends at $r=0.168 m$ below which cobs are attached to the discs. Hence the cavity radius ratio and gap ratio are 0.51 and 0.31 respectively. An approximate profile was chosen for the cob geometry. The equispaced radial vanes inbetween the cobs, which were a part of Farthing et al.'s [11] experiments, were included only in R2.2. In the axisymmetric model, the inlet was approximated as a small circumferential slot with an area equal to the area of intersection of nozzles with the shroud.

The simulations were carried out in the rotating frame of reference. Total pressure and temperature at the inlet were assumed to be $101300 P a$ and $288 \mathrm{~K}$. Velocity angles were specified at the inlet in the axisymmetric modelling. In $3 D$ modelling of case $R 2.1$, the flow was assumed to enter the outer settling chamber through a narrow slot with an inlet swirl of $c=1$. In $R 2.2$, uniform total pressure and temperature are specified at inlet with inflow parallel to nozzle axes. To achieve the desired mass flow rate through the cavity in the Hydra solver the exit pressure was varied keeping constant the total pressure and temperature at the inlet. In the FLUENT simulations a 'mass flow inlet' boundary condition was used and the static pressure at the outlet was adjusted so that the total pressure at the inlet was close to 101300 $\mathrm{Pa}$ in the converged solution.

In the axisymmetric modelling of case R2.1, the mesh dependency was checked by computing the solutions on two meshes with 105,000 and 160,000 hexahedral cells. The results showed mesh independent solutions. However, the difference between the sum of angular momentum flux at inlet and outlet and the sum of wall torques was considerably higher for this test case, being upto $0.007 \mathrm{Nm}$. This angular momentum imbalance was around $8 \%$ of the "free disc" moment (Chew [31]) for a similar disc, for which the expected moment is approximately $0.083 \mathrm{Nm}$. $3 D$ simulations were undertaken using the Hydra solver as explained above. Mesh dependency was checked by computing the solutions on two grids with 1.61 million and 860,000 hexahedral cells. The solutions on these two meshes confirmed mesh independent results. The angular momentum balance check showed that the difference between the sum of inlet and outlet angular momentum flux and wall torques was less than $0.0001 \mathrm{Nm}$. Thus the $3 \mathrm{D}$ simulations considerably improved the angular momentum balance in the cavity compared to the axisymmetric case.

The flow conditions used for Test case $R 2$ are tabulated in Table 1. In case R2.2 the simulations were carried on a mesh with 307,000 hexahedral cells. It is interesting to note that omission of settling chamber and inclusion of a radial vane in the sector below $x<0.5$ for this case reduced the mesh size considerably without affecting the solutions inside the cavity.

5.2.2 Results: Case R2.1 Axisymmetric computations with wall functions were performed using both the eddy viscosity models in the Hydra and FLUENT solvers. The RSM model in FLUENT was also used for axisymmetric modelling of the test case. For $3 D$ computations the SA and $k-\varepsilon$ models in the Hydra solver were used. Since the inlet swirl fraction was less than 1.0, radial outflow in the outer part of the cavity and stagnation points on both the discs were observed. Hence, lower $y^{+}$values were inevitable near the discs and were around 10 to 20 near the stagnation points. The pressure measurements were taken on the left hand disc of the cavity shown in Fig 9.

\begin{tabular}{||c|c|c|c||}
\hline Case & $\mathbf{C}_{\mathbf{w}}$ & $\mathbf{c}$ & $\lambda_{t}$ \\
\hline \multirow{4}{*}{$\boldsymbol{R} 2.1$} & 3050 & 0.4 & 0.072 \\
\cline { 2 - 4 } & 5080 & 0.0 & 0.120 \\
\hline \hline \multirow{4}{*}{$\boldsymbol{R} 2.2$} & 950 & 0.9 & 0.022 \\
\cline { 2 - 4 } & 2030 & 0.8 & 0.048 \\
\cline { 2 - 4 } & 4070 & 0.6 & 0.095 \\
\cline { 2 - 4 } & 6090 & 0.4 & 0.143 \\
\cline { 2 - 4 } & 8100 & 0.2 & 0.190 \\
\cline { 2 - 4 } & 10100 & 0.0 & 0.238 \\
\hline
\end{tabular}

TABLE 1. Flow parameters considered for test case $R 2\left(R e_{\phi}=\right.$ $\left.0.61 \times 10^{6}\right)$. 

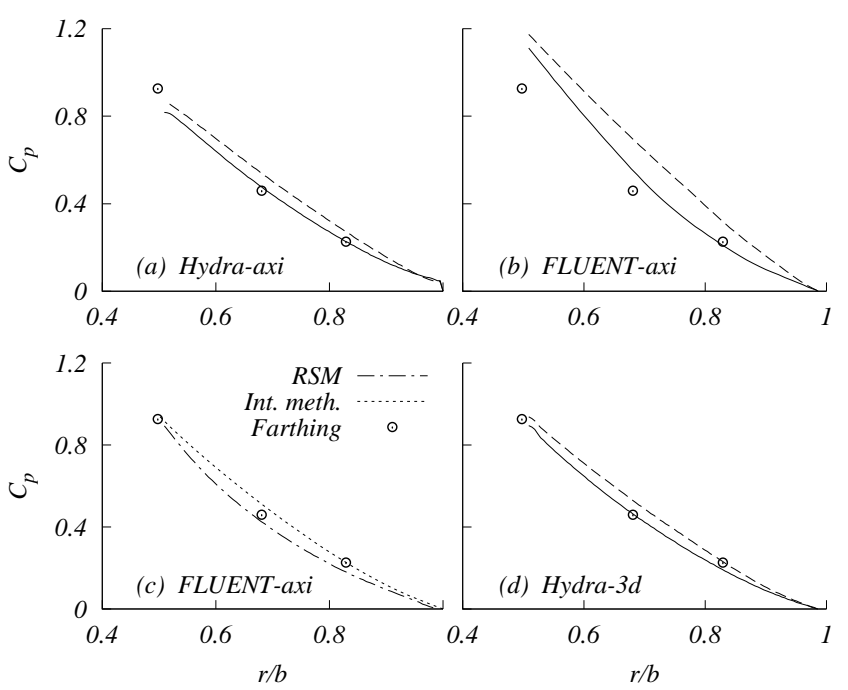

FIGURE 10. Non-dimensional pressure distributions on the left hand disc for the test case R2.1 with $\operatorname{Re}_{\phi}=2.1 \times 10^{6}, c=0.4$ and $\lambda_{t}=0.072$. Results from — SA model, - - - k-E model, 'o' Farthing's [14] experiments

For the axisymmetric case with $c=0.4$, the solutions obtained from the eddy viscosity models in the Hydra solver matched well with the measurements. This is shown in Fig 10(a). Where as the axisymmetric results from the FLUENT's eddy viscosity models showed higher pressure drop than the measurements at the lower radius of the cavity as shown in Fig 10(b). $C_{p}$ predicted by axisymmetric simulation in RSM model and the integral method (Fig 10(c)) were in close agreement with the measurements . The $C_{p}$ distributions obtained from the $3 D$ simulations in Hydra are shown in Fig 10(d). As for the axisymmetric model, both the eddy viscosity models showed good agreement with the measurements.

When the inlet de-swirl was increased for the second test case, with $c=0$, the SA model in both the solvers showed lower pressure distribution than the measurements in the axisymmetric simulations. Results from the axisymmetric simulations in Hydra and FLUENT for the $c=0$ case are shown in Fig 11 (a) and (b) respectively. Predictions from the axisymmetric simulations in RSM agreed well with the measurements as shown in Fig 11(c). The integral method predicted slightly higher pressure drop than the experiments. Fig 11 (d) shows $C_{p}$ predicted by the $3 D$ simulations in Hydra for the same case. In the $3 D$ simulations also the SA model predicted lower pressures as shown in Fig 11 (c). The $k-\varepsilon$ model results from axisymmetric and $3 D$ computations matched fairly well with the experimental results.

5.2.3 Results: Case R2.2 3D simulations were performed in FLUENT using $k-\varepsilon$ and RSM models with standard wall functions. The mass flow rate was controlled at the inlet to achieve the desired $C_{w}$ values, as explained above. The pressure coefficients at $r=a\left(C_{p, a}\right)$ predicted by two turbulence models
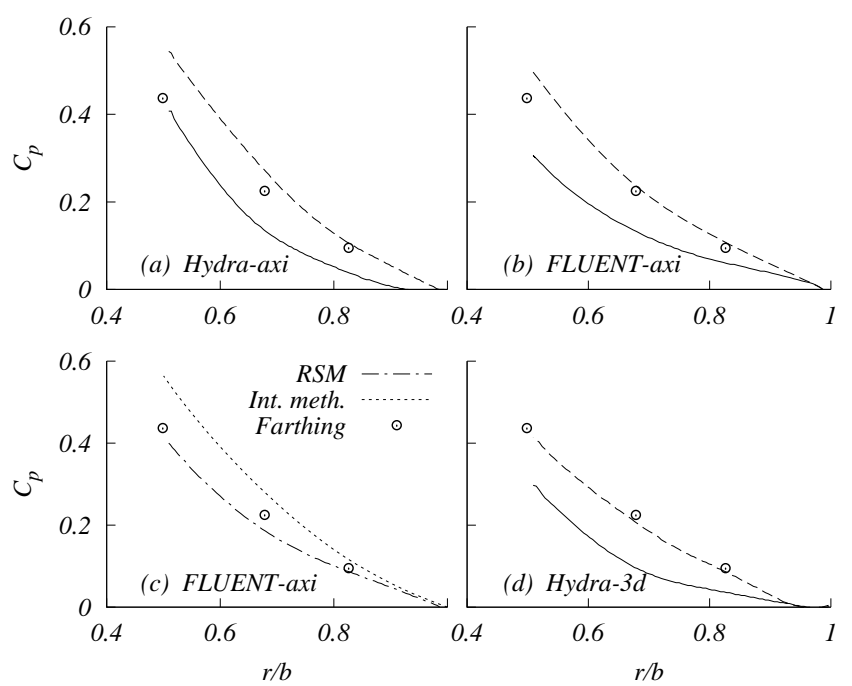

FIGURE 11. Non-dimensional pressure distributions on the left hand disc for the test case R2.1 with $R e_{\phi}=2.1 \times 10^{6}, c=0.0$ and $\lambda_{t}=0.120$. Results from — SA model, - - - k- $\varepsilon$ model, 'o' Farthing's [11, 14] experiments

and the integral method are shown in Fig 12 and compared with measurements. Corresponding inlet swirl fractions $(c)$ are tabulated in Table 1. When the inlet swirl was reduced from $c=1$, the measured $C_{p, a}$ in the cavity increased and reached a maximum value at around $\left(C_{w} \approx 2000, c \approx 0.8\right)$. Further reduction in inlet swirl resulted in reduction of $C_{p, a}$. Both the turbulence models and the integral method reproduced this overall behaviour reasonably well. Among the CFD results, the predictions from RSM were closer to the measurements when $c>0.7$ and $k-\varepsilon$ results were closer for lower values of inlet swirl $(0.2<c<0.7)$.

The radial velocity contours on the radial-axial plane, midway between two successive nozzle exits, are shown in Fig 13. Interestingly, when the inlet swirl is higher (Fig 13 (a \& b)), the flow in the cavity is almost symmetric on either side of the axial mid-plane which is also an assumption in deriving the integral theorem equations. Most of the radial inflow occurs near to the discs in these cases and hence the integral theorem solutions agree well with the measurements. For $c<0.5$, the flow is asym-

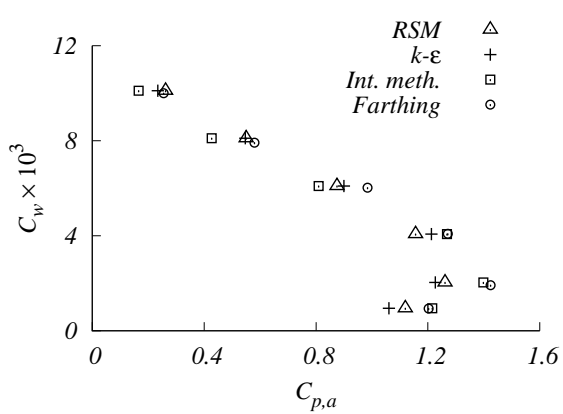

FIGURE 12. Variation of $C_{p, a}$ with respect to $C_{w}$ in the cavity in test case $R 2.2\left(R e_{\phi}=0.61 \times 10^{6}\right)$

Copyright (c) 2012 by Rolls-Royce plc. 


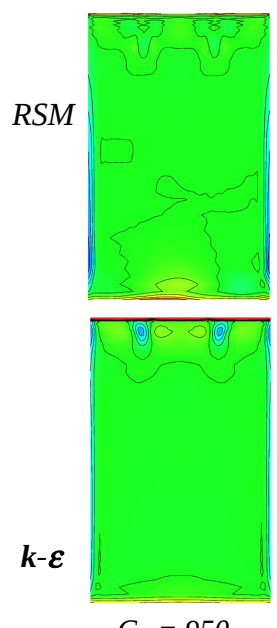

$C_{w}=950$

(a) $c=0.9$
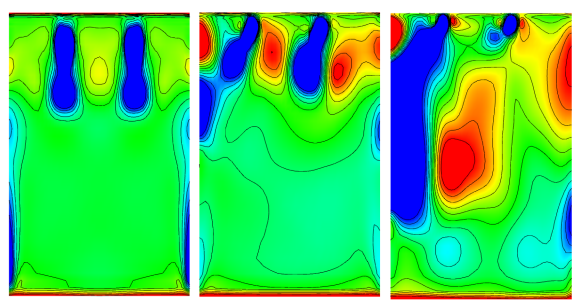

$u, m / s$

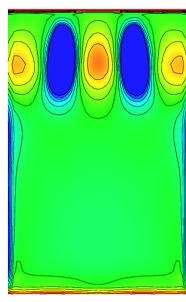

$C_{w}=4070$

(b) $c=0.6$

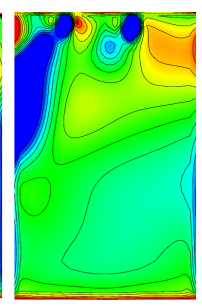

$C_{w}=6090$

(c) $c=0.4$

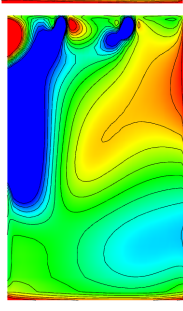

$C_{w}=8100$

(d) $c=0.2$

FIGURE 13. Radial velocity contours on the radial-axial plane, midway between two successive nozzle exits in test case R2.2

metrical and the inflow is higher near the left hand disc. Since the integral method does not account for this unequal radial inflow near the discs it is less accurate at lower values of $c$. For these conditions turbulence models better match the measurements.

An unsteady simulation was carried out for a duration of approximately 60 sector passings ( 23 full cavity rotations) for the case with $c=0.2$ using RSM turbulence model. This showed no significant change in flow structure. There were, however, slight flow fluctuations in the $\phi$ direction, especially in a thin layer near the shroud. In the RSM model the total torque imbalance or unsteady fluctuation was nearly $3 \%$ of the sum of cavity disc torques. Further, both the models showed nearly similar flow features in the cavity.

\subsection{Test case R3}

Heat transfer measurements in a rotating cavity similar to that of test case R2 were reported by [14]. The inlet was modified by introducing a foam filled slot in the mid axial plane of the shroud thus giving the inlet swirl of $c=1$. The cavity dimensions are shown in Fig 9, the only modification being the outer radius was increased to $b=0.391 \mathrm{~m}$ from $0.33 \mathrm{~m}$. The outer surface of the left hand disc, shown in Fig 9, is heated by an external radiant heater. The right hand disc, the shroud and the cobs were thermally insulated. Temperature distributions on the inner and outer surfaces of the heated disc were measured using thermocouples. Flux meters were used to estimate the heat flux. In addition to this, using the measured temperatures, the transient Fourier heat equation was solved to estimate the heat flux through the heated disc. The local Nusselt numbers on the disc surface were reported for various flow and rotational Reynolds numbers.

5.3.1 Formulation For CFD modelling, the case with $R e_{\phi}=1.2 \times 10^{6}$ and $C_{w}=3500$ was considered. A $1^{\circ}$ sector
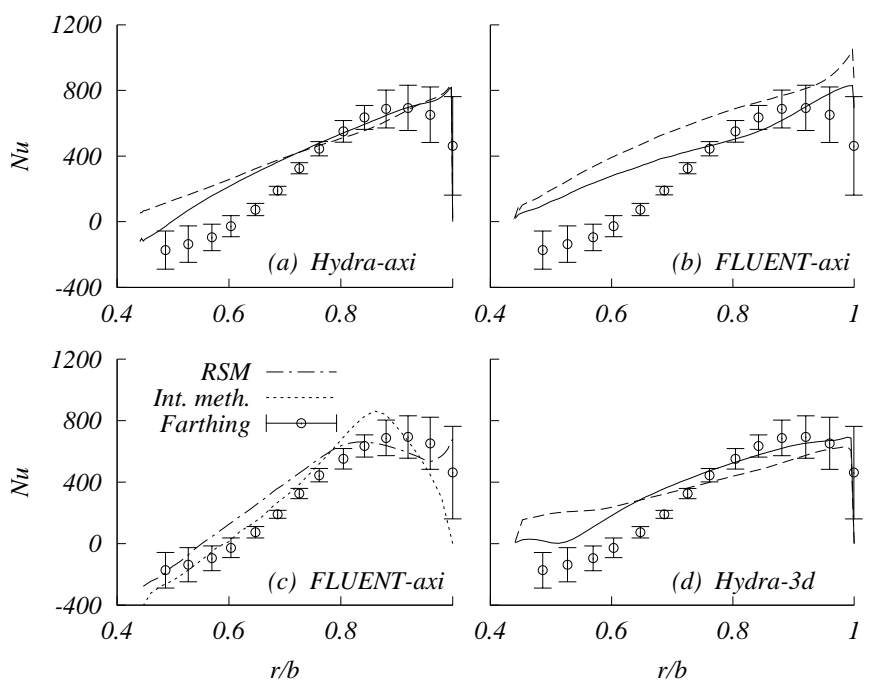

FIGURE 14. Local Nusselt number on the left hand disc for the test case $R 3$ with $R_{\phi}=1.2 \times 10^{6}, C_{w}=3500$ and $c=1.0$. - SA model, - - - k- $\varepsilon$ model, 'o' Farthing's [14] experiments

was selected for axisymmetric simulations and an $18^{\circ}$ sector was considered for the $3 \mathrm{D}$ simulations. The radial vanes in-between the cobs were included in the 3D domain. Total pressure and temperature at the inlet were assumed to be $101300 \mathrm{~Pa}$ and $298 \mathrm{~K}$ respectively. All the walls except the left hand disc were assumed to be adiabatic. The temperature profile obtained from the measurements was specified on the left hand disc. The fine mesh and the coarse mesh considered for the axisymmetric modelling had 64,000 and 30,000 hexahedral cells respectively, and the solutions were found to be reasonably mesh independent. The maximum difference between local Nusselt numbers on these meshes at any location was less than $1.04 \%$ of the peak value. The mesh considered for 3D simulations had 228, 000 hexahedral cells. This test case had considerably fewer cells than test case R2 because the inlet slot was $10 \mathrm{~mm}$ wide compared to the $0.8 \mathrm{~mm}$ wide slot in test case R2. For the 3D modelling, there was no need to model the nozzle and settling chamber as in test case R2 thus keeping the number of mesh points significantly less. $3 \mathrm{D}$ simulations were attempted only with the Hydra solver. The overall angular momentum imbalance (the difference between change in angular momentum flux of the fluid in the cavity and the total wall torque) was less than $1.5 \%$ of the total change in angular momentum flux in the cavity for all the computations. The energy imbalance was within $2 \%$ of the difference in inlet and outlet energy flux and the work done on the fluid for the SA model and around $4 \%$ for the $k-\varepsilon$ model.

5.3.2 Results Local Nusselt numbers were calculated using the relation $N u=q_{0} r /\left(k\left(T_{0}-T_{o}\right)\right)$, where $q_{0}$ is the surface heat flux on the left hand disc, $k$ is the thermal conductivity of air, $T_{0}$ is the disc temperature at radius $r$ and $T_{o}$ is the total temperature of air at the inlet. The local Nussult numbers on the 
heated disc computed using the SA model with wall functions, shown in Fig 14(a \& b), agreed well with the measurements for $r / b>0.75$ and predicted higher heat transfer than measured below this radius. Little difference was found between axisymmetric and 3D model results shown in Fig 14(d). The agreement with the measurements was somewhat worse for the $k-\varepsilon$ model. The $k$ - $\varepsilon$ model in FLUENT showed consistently higher heat transfer rates than the experiments at all radii as shown in Fig 14(b). The integral method showed a reasonable match with the experimental results at all radii. The RSM model showed the closest agreement with the measurements among all the tested models as shown in Fig 14(c). The RSM model also predicted the reversal of heat transfer from the fluid to the disc at $r / b<0.56$ which was reported in the experiments close to this radial location.

\section{Conclusions}

Numerical investigation of flow and heat transfer in stationary and rotating cylindrical cavities with superimposed radial inflow have been carried out. Two eddy viscosity models, $k-\varepsilon$ and SA, a Reynolds stress model and an integral method have been used to evaluate the flow and heat transfer. For selected test cases eddy viscosity models were tested in two different codes. Numerical predictions have been evaluated against previously published flow and heat transfer measurements for narrow and wide vortex chambers and rotating cavities with swirled and deswirled inflows. The eddy viscosity and integral methods are representative of industrial practice while the RSM is often proposed as a more suitable model for highly rotating flows.

Of all the models tested the RSM gave best agreement with the measurements. Results obtained using this model agreed reasonably well with the flow and heat transfer data for all the test cases.

The two eddy viscosity models tested showed mixed levels of agreement with the measurements. Both $k-\varepsilon$ and SA models gave poor results for a wide vortex chamber. This is attributed to over-prediction of turbulent diffusion in the rotating core by these models. This shortcoming also affected results for the narrow vortex chamber with the $k-\varepsilon$ model showing particularly poor predictions in the central flow region where the swirl velocity is particularly strong.

For the rotating cavity cases both the models might be said to have given reasonable estimates of flow and heat transfer, but SA gave slightly better than the $k-\varepsilon$ model overall. This indicates that in cases where turbulent diffusion given by the models has little effect in the core flow region, reasonable results are obtained. This is consistent with industrial acceptance of these models for many turbomachinery flows.

Both the eddy viscosity models showed some sensitivity to solvers when using wall functions. Wall function implementation in the respective solvers is considered to be the reason for these differences. This is supported by tests showing good agreement between the two codes when near-wall resolved modelling is used.

Results from the integral method generally agreed very well with the measurements. This model neglects any turbulent diffusion in the central core flow, avoiding shortcomings by the eddy viscosity models in these regions. The success of the integral method suggests some insensitivity to the boundary layer model. The eddy viscosity models do, however, show some sensitivity to the wall function implementation, as has been observed for other disc cavity flows.

\section{ACKNOWLEDGMENT}

This research was funded by Rolls-Royce plc and the Engineering and Physical Science Research Council through a Dorothy Hodgkin Postgraduate Award. The authors are greatful to useful comments and advice given by colleagues at the University of Surrey and at Rolls-Royce plc.

\section{REFERENCES}

[1] Owen, J. M., and Wilson, M., 2001. "Some Current Research in Rotating-disc Systems". Annals New York Academy of Sciences, Heat Transfer in Gas Turbine Systems, 934, pp. 206-221.

[2] Owen, J. M., Pincombe, J. R., and Rogers, R. H., 1985. "Source-sink Flow Inside a Rotating Cavity". J. Fluid Mech., 155, pp. 233-265.

[3] Hide, R., 1968. "On Source-sink Flows in a Rotating Fluid”. J. Fluid Mech., 32, pp. 737-764.

[4] Wormley, D. N., 1969. "An Analytical Model for the Incompressible Flow in Short Vortex Chambers". ASME J. Basic Engg., pp. 264-272.

[5] Chew, J. W., and Snell, R. J., 1988. "Prediction of the Pressure Distribution for Radial Inflow Between Co-rotating Discs". ASME GT and Aeroengine Congress, Amsterdam,, Paper No 88-GT-61, p. 9.

[6] Owen, J. M., and Rogers, R. H., 1995. "Flow and Heat Transfer in Rotating-disc Systems, volume 2: Rotating cavities". Mechanical Engineering Research Studies, Engineering Design Series, 2.

[7] Shevchuk, I. V., 2009. "Convective Heat and Mass Transfer in Rotating Disk Systems". Lecture Notes in Applied and Computational Mechanics, $\mathbf{4 5}$.

[8] Childs, P. R. N., 2010. "Rotating flows". ButterworthHeinemann, ISBN:9780123820983.

[9] Firouzian, M., Owen, J. M., Pincombe, J. R., and Rogers, R. H., 1985. "Flow and Heat Transfer in a Rotating Cavity with a Radial Inflow of Fluid. Part 1: The Flow Structure". Int. J. Heat and Fluid Flow, 6(4), pp. 228-234.

[10] Firouzian, M., Owen, J. M., Pincombe, J. R., and Rogers, R. H., 1986. "Flow and Heat Transfer in a Rotating Cavity with a Radial Inflow of Fluid. Part 2: Velocity, Pressure and Heat Transfer Measurements". Int. J. Heat and Fluid Flow, 7(1), pp. 21-27. 
[11] Farthing, P. R., Chew, J. W., and Owen, J. M., 1991. "The Use of De-swirl Nozzles to Reduce the Pressure Drop in a Rotating Cavity with a Radial Inflow". J. Turbomach., 113(1), pp. 106-114.

[12] Chew, J. W., Farthing, P. R., Owen, J. M., and Stratford, B., 1989. "The Use of Fins to Reduce the Pressure Drop in a Rotating Cavity with a Radial Inflow". J. Turbomach., 111(3), pp. 349-356.

[13] Volchkov, E. P., Semenov, S. V., and Terekov, V., 1991. "Heat Transfer and Shear Stress at the End Wall of a Vortex Chamber". Experimental Thermal and Fluid Science, 4(5), pp. 546-557.

[14] Farthing, P. R., 1989. "The Effect of Geometry on Flow and Heat Transfer in a Rotating Cavity". D.Phil Thesis, University of Sussex.

[15] Morse, A. P., 1988. "Numerical Prediction of Turbulent Flow in Rotating Cavities". ASME J. Turbomach., 110, pp. 202-211.

[16] Young, C., and Snowsill, G. D., 2003. "CFD Optimization of Cooling Air Offtake Passages Within Rotor Cavities". $J$. Turbomach, 125(2), pp. 380-386.

[17] Gosman, A. D., Lockwood, F. C., and Loughhead, J. N., 1976. "Prediction of Recirculating, Swirling Flow in Rotating Disc Systems". J. Mech. Eng. Sci., 18(3), pp. 142-148.

[18] Chew, J. W., 1984. "Prediction of Flow in Rotating Disc Systems Using the $k-\varepsilon$ Turbulence Model". ASME Gas Turbine Conf., Amsterdam, ASME 84-GT-229.

[19] Dacles-Mariani, J., Zilliac, G. G., Chow, J. S., and Bradshaw, P., 1995. "Numerical/Experimental Study of a Wingtip Vortex in the Near Field". AIAA Journal, 33(9), pp. 1561-1568.

[20] Spalart, P. R., and Shur, M., 1997. "On the Sensitization of Turbulence Models to Rotation and Curvature". Aerospace Science and Technology, 1(5), pp. 297-302.

[21] Spalart, P. R., and Allmaras, S. R., 1994. "A One-equation Turbulence Model for Aerodynamic Flows". La Recherche Aerospatiale, 1, pp. 5-21.

[22] Torii, S., and Yang, W. J., 1995. "Numerical Prediction of Fully Developed Turbulent Swirling Flows in an Axially Rotating Pipe by Means of a Modified k- $\varepsilon$ Turbulence Model". Int. J. Num. Meth. Heat Fluid Flow, 5(2), pp. 175183.

[23] Smirnov, P. E., and Menter, F. R., 2009. "Sensitization of the sst Turbulence Model to Rotation and Curvature by Applying the spalart shur Correction Term". J. Turbomach., 131(4), p. 8.

[24] Iacovides, H., and Toumpanakis, P., 1993. "Turbulence Modeling of Fows in Axisymmetric Rotor-stator Systems". Proc. 5th Int. Symp. Refined Flow Modeling Turbulence Measurements, Paris: Int. Assoc. Hydraul. Res., p. 835.

[25] Elena, L., and Schiestel, R., 1996. "Turbulence Modeling of Rotating confined flows". Int. J. Heat and Fluid Flow,
17, pp. 283-289.

[26] Chen, J. C., and Lin, C. A., 1999. "Computations of Strongly Swirling Flows with Second-moment Closures". Int. J. Num. Meth. in Fluids, 30(5), pp. 493-508.

[27] Virr, G. P., Chew, J. W., and Coupland, J., 1994. "Application of Computational Fluid Dynamics to Turbine Disc Cavities". J. Turbomach., 116(4), pp. 701-708.

[28] Soghe, R. D., Innocenti, L., Andreini, A., and Poncet, S., 2010. "Numerical Benchmark of Turbulence Modeling in Gas Turbine Rotor-stator System". Proc. of ASME Turbo Expo 2010: Power for Land Sea \& Air, GT2010, 7, pp. 771783.

[29] Launder, B. E., and Spalding, D. B., 1974. "The Numerical Computation of Turbulent Flows". Computer Methods in Applied Mechanics and Engineering, 3(2), pp. 269-289.

[30] Karman, von. Th., 1924. "Uber Laminare und Turbulente Reibung". ZAMM - Journal of Applied Mathematics and Mechanics, 1(4), pp. 233-252.

[31] Chew, J. W., 1987. "Computation of Flow and Heat Transfer in Rotating Disc Systems". Proceedings of the 2nd ASME-JSME Thermal Engineering Conference, Hawaii,, 3, pp. 361-367.

[32] Chew, J. W., and Rogers, R. H., 1988. “An Integral Method for the Calculation of Turbulent Forced Convection in a Rotating Cavity with Radial Outflow". Int. J. Heat and Fluid Flow, 9(1), pp. 37-48.

[33] May, N. E., Chew, J. W., and James, P. W., 1994. "Calculation of Turbulent Flow for an Enclosed Rotating Cone". ASME J. Turbomach, 116(3), pp. 548-554.

[34] Moinier, P., 1999. "Algorithm Developments for an Unstructured Viscous Flow Solver". D.Phil thesis, University of Oxford. UK.

[35] FLUENT, 2006. "Fluent 6.3 documentation". ANSYS, Inc.

[36] Javiya, U., Chew, J. W., Hills, N. J., Zhou, L., Wilson, M., and Lock, G. D., 2011. "CFD Analysis of Flow and Heat Transfer in a Direct Transfer Preswirl System”. J. Turbomach., 134(3).

[37] Howard, J. H. G., Patankar, S. V., and Bordynuik, R. M., 1980. "Flow Prediction in Rotating Ducts Using coriolismodified Turbulence Models". ASME J. Fluids Eng., 102, pp. 456-461.

[38] Shur, M. L., Strelets, M. K., and Travin, A. K., 2000. “Turbulence Modelling in Rotating and Curved Channels: Assessing the spalart-shur Correction". AIAA Journal, 38(5), pp. 784-792.

[39] Iaccarino, G., Ooi, A., Reif, B. A. P., and Durbin, P., 1999. "RANS Simulations of Rotating Flows". Center for Turbulence Research, Annual Research Briefs.

[40] Poncet, S., Soghe, R. D., and Facchini, B., 2010. "Rans Modelling of Flow in Rotating Cavity System". V European Conference on Computational Fluid Dynamics ECCOMAS CFD. 